\title{
Reduced brain UCP2 expression mediated by microRNA-503 contributes to increased stroke susceptibility in the high-salt fed stroke-prone spontaneously hypertensive rat
}

\author{
Speranza Rubattu ${ }^{\star, 1,2}$, Rosita Stanzione ${ }^{2}$, Franca Bianchi ${ }^{2}$, Maria Cotugno ${ }^{2}$, Maurizio Forte ${ }^{2}$, Floriana Della Ragione ${ }^{2,3}$, \\ Salvatore Fioriniello ${ }^{3}$, Maurizio D'Esposito, ${ }^{2,3}$, Simona Marchitti ${ }^{2}$, Michele Madonna ${ }^{2}$, Simona Baima ${ }^{4}$, Giorgio Morelli ${ }^{4}$, \\ Sebastiano Sciarretta ${ }^{2,5}$, Luigi Sironi, ${ }^{6,7}$, Paolo Gelosa ${ }^{7}$ and Massimo Volpe ${ }^{1,2}$
}

UCP2 maps nearby the lod score peak of STR1-stroke QTL in the SHRSP rat strain. We explored the potential contribution of UCP2 to the high-salt diet (JD)-dependent increased stroke susceptibility of SHRSP. Male SHRSP, SHRSR, two reciprocal SHRSR/SHRSPSTR1/QTL stroke congenic lines received JD for 4 weeks to detect brain UCP2 gene/protein modulation as compared with regular $\operatorname{diet}(\mathrm{RD})$. Brains were also analyzed for NF- $\kappa \mathrm{B}$ protein expression, oxidative stress level and UCP2-targeted microRNAs expression level. Next, based on knowledge that fenofibrate and Brassica Oleracea (BO) stimulate UCP2 expression through PPAR $\alpha$ activation, we monitored stroke occurrence in SHRSP receiving JD plus fenofibrate versus vehicle, JD plus BO juice versus BO juice plus PPAR $\alpha$ inhibitor. Brain UCP2 expression was markedly reduced by JD in SHRSP and in the (SHRsr.SHRsp-(D1Rat134Mt1pa)) congenic line, whereas NF- $\kappa$ B expression and oxidative stress level increased. The opposite phenomenon was observed in the SHRSR and in the (SHRsp.SHRsr-(D1Rat134-Mt1pa)) reciprocal congenic line. Interestingly, the UCP2-targeted rno-microRNA-503 was significantly upregulated in SHRSP and decreased in SHRSR upon JD, with consistent changes in the two reciprocal congenic lines. Both fenofibrate and BO significantly decreased brain microRNA-503 level, upregulated UCP2 expression and protected SHRSP from stroke occurrence. In vitro overexpression of microRNA-503 in endothelial cells suppressed UCP2 expression and led to a significant increase of cell mortality with decreased cell viability. Brain UCP2 downregulation is a determinant of increased stroke predisposition in high-salt-fed SHRSP. In this context, UCP2 can be modulated by both pharmacological and nutraceutical agents. The microRNA-503 significantly contributes to mediate brain UCP2 downregulation in JD-fed SHRSP.

Cell Death and Disease (2017) 8, e2891; doi:10.1038/cddis.2017.278; published online 22 June 2017

The SHRSP represents a suitable animal model for the investigation of the etiopathogenetic basis of hypertensive target organ damage. ${ }^{1}$ Feeding SHRSP with JD accelerates both renal and cerebrovascular damage occurrence ${ }^{2,3}$ with renal damage preceding stroke.,4,5 The gene encoding UCP2 maps nearby the lod score peak of STR1/stroke QTL identified on rat chromosome 1 in the SHRSP. $^{3}$ UCP2 is a inner mitochondrial membrane protein that exerts an antioxidant effect in various tissues by regulating fatty acid oxidation, mitochondrial biogenesis, substrate utilization and ROS elimination, ${ }^{6}$ and is regulated by PPARa. ${ }^{7}$ The latter, a member of nuclear receptor family of ligand-activated transcription factors, is known to regulate lipid and energy metabolism through the uncoupling proteins; ${ }^{7}$ it also exerts anti-inflammatory and antioxidant effects in many cell types, including cardiovascular cells. ${ }^{8}$
UCP2 downregulation associates with increased oxidative stress, atherosclerosis, vascular damage and shorter lifespan in mice. ${ }^{9-12}$ UCP2 overexpression significantly prevented ROS production in endothelial cells and preserved endothelial function by reducing ROS levels. ${ }^{13,14}$ Consistently with its ability to decrease endogenous mitochondrial ROS production and to maintain normal mitochondrial membrane potential and ATP levels, a neuroprotective effect of UCP2 has been previously described both in vitro and in vivo. ${ }^{15-21} \mathrm{We}$ previously reported an age-related spontaneous decrease of UCP2 gene and protein expression only in the brain of SHRSP, preceding spontaneous stroke occurrence at 1 year of age. ${ }^{22}$

Of interest, we have shown that, in association with increased renal injury, JD significantly downregulates UCP2 gene and protein expression in the kidneys of SHRSP, but not of SHRSR. ${ }^{23}$ Consistent findings were obtained in the kidneys of SHRSR/SHRSP-derived stroke congenic lines, depending

\footnotetext{
${ }^{1}$ Department of Clinical and Molecular Medicine, School of Medicine and Psychology, Sapienza University of Rome, Ospedale S. Andrea, Rome, Italy; ${ }^{2}$ Istituto di Ricovero e Cura a Carattere Scientifico (IRCCS) Neuromed, Località Camerelle, Pozzilli, Italy; ${ }^{3}$ Institute of Genetics and Biophysics 'A. Buzzati-Traverso', Naples, Italy; ${ }^{4}$ Food and Nutrition Research Center (CRA-NUT), Consiglio per la Ricerca in agricoltura e l'analisi dell'economia agraria, Rome, Italy; ${ }^{5}$ Department of Medical-Surgical Sciences and Biotechnologies, Sapienza University of Rome, Latina, Italy; ${ }^{6}$ Department of Pharmacological and Biomolecular Sciences, University of Milan, Milan, Italy and ${ }^{7}$ Centro Cardiologico Monzino IRCCS, Milan, Italy

${ }^{*}$ Corresponding author: S Rubattu, Clinical and Molecular Medicine Department, School of Medicine and Psychology, Sapienza University, S.Andrea Hospital, IRCCS Neuromed, Pozzilli (Is), Rome, Italy. Tel: +39 06 33775979; Fax: +39 06 33775061; E-mail: rubattu.speranza@neuromed.it

Received 15.2.17; revised 27.4.17; accepted 16.5.17; Edited by A Verkhratsky
} 
on the genetic configuration of the transferred UCP2. ${ }^{24}$ In vitro, $U C P 2$ silencing in renal mesangial cells led to increased inflammation, oxidative stress and cell mortality. ${ }^{23}$ Exposure of primary renal proximal tubular epithelial cells isolated from SHRSP to high- $\mathrm{NaCl}$ medium led to UCP2 downregulation and reduced viability, which was rescued by recombinant

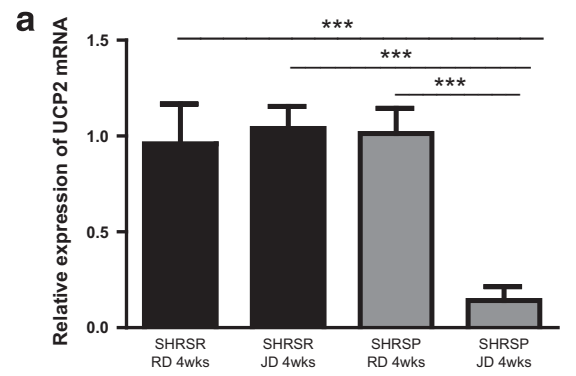

b

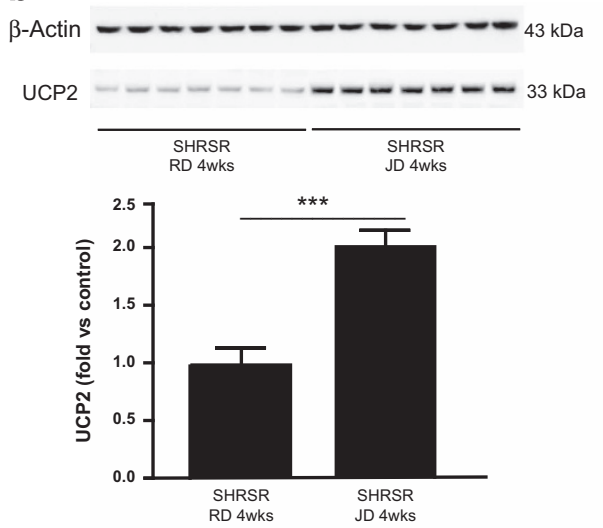

d

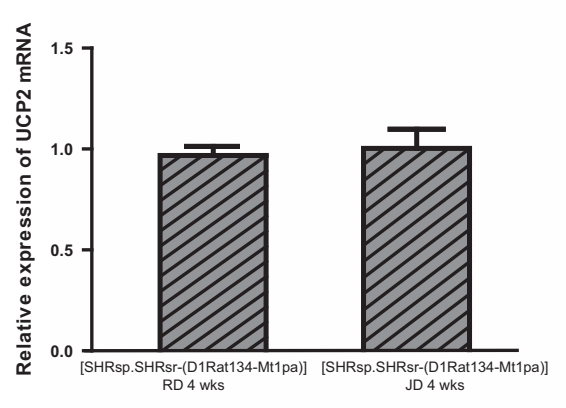

f

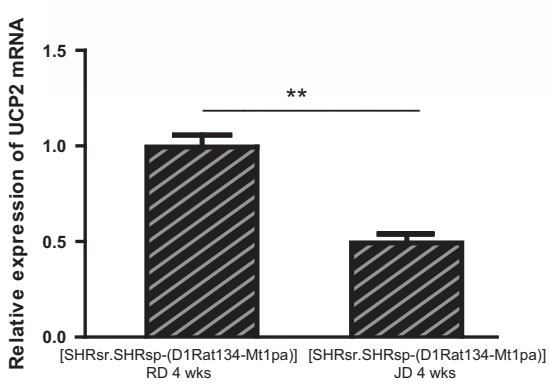

C

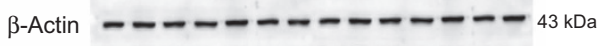

UCP2
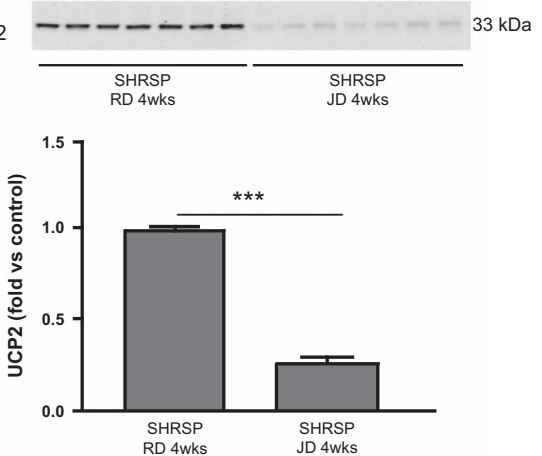

e

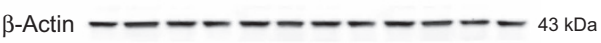
$\mathrm{UCP} 2----=---\infty \mathrm{kDa}$ [SHRsp.SHRsr-(D1Rat134-Mt1 pa)]
[SHRsp.SHRsr-(D1Rat134-Mt1pa)]
JD 4 wks

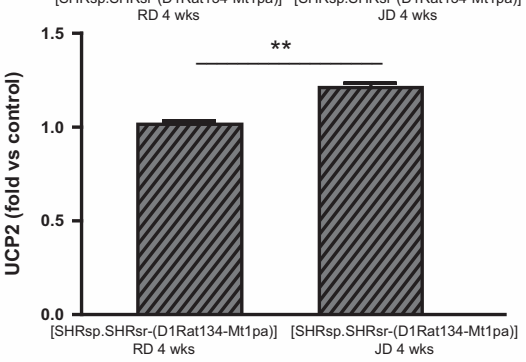

g

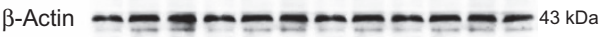
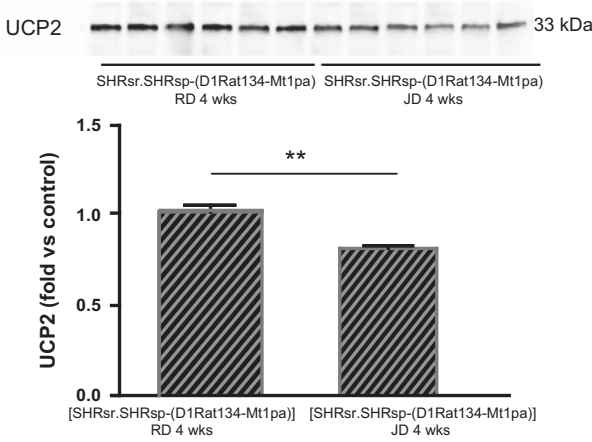
UCP2. ${ }^{24}$ Moreover, the PPAR $a$-mediated upregulation of UCP2 gene and protein expression by $\mathrm{BO}$ sprouts juice, administered along with JD, completely prevented renal damage occurrence in SHRSP. ${ }^{25}$ As expected, the selective inhibition of PPARa reduced the beneficial effects of BO on the renal injury of this strain. ${ }^{25}$

Notably, fenofibrate, a compound that exerts renal and neuroprotection in various experimental settings through its impact on several antioxidant enzymes, ${ }^{26-28}$ and that is also known to stimulate PPAR $a$ and UCP2 expression, ${ }^{29}$ promoted protection from target organ damage in SHRSP. ${ }^{30}$

Based on the above-mentioned observations, the aims of the present study were: (1) to assess for the first time the modulation of UCP2 in the brain of high-salt-fed SHRSP versus SHRSR, as well as in two SHRSR/SHRSP-STR1/QTL stroke congenic lines; (2) to explore the impact of PPAR $a$ and UCP2 expression modulation by $\mathrm{BO}$ and fenofibrate on the stroke susceptibility of high-salt-fed SHRSP; and (3) to explain part of the mechanisms underlying brain UCP2 downregulation upon JD in the stroke-prone strain.

\section{Results}

Impact of 4 weeks JD feeding on brain UCP2 expression and related inflammatory and oxidative stress parameters in the four rat lines. Four weeks of JD feeding induced a significant UCP2 gene and protein expression downregulation only in the SHRSP brain (Figures $1 \mathrm{a}-\mathrm{c}$ ), as previously reported in the kidneys. ${ }^{23-25}$ The (SHRsp.SHRsr(D1Rat134-Mt1pa)) congenic line, derived from the SHRSP parental strain and carrying the SHRSR/STR1 chromosomal fragment, did not downregulate UCP2 under JD, differently from the SHRSP strain of origin (Figures 1d and e). Vice versa, the (SHRsr.SHRsp-(D1Rat134-Mt1pa)) congenic line, derived from the SHRSR parental strain and carrying the SHRSP/STR1 chromosomal fragment, significantly downregulated UCP2 under JD, differently from the SHRSR strain of origin (Figures $1 \mathrm{f}$ and $\mathrm{g}$ ). These results confirmed the key role of UCP2 configuration (SP or SR) for the response to high-salt diet.

Figure 2 shows the NF- $k$ B protein expression level, a marker of inflammation, and the carbonylated protein level, a marker of oxidative stress, in the brains of the parental lines (SHRSR: panels a and b; SHRSP: panels $c$ and d). Both inflammatory and oxidative stress markers were significantly increased only in the brains of JD-fed SHRSP. Figure 3 shows the same parameters in the two STR1/QTL stroke congenic lines, the one derived from the SHRSP (panels $a$ and $b$ ), and the one derived from the SHRSR (panels $c$ and d). Both inflammatory and oxidative stress markers were decreased in the
SHRSP-derived stroke congenic line, carrying the SHRSR/ STR1 chromosomal fragment (panels a and b), whereas these markers increased significantly in the SHRSR-derived stroke congenic line carrying the SHRSP/STR1 chromosomal fragment (panels $c$ and d). These results confirmed that, whenever UCP2 expression was downregulated, such as in JD-fed SHRSP and JD-fed (SHRsr.SHRsp-(D1Rat134-Mt1pa)), the degree of inflammation and of oxidative stress increased. Vice versa, no increase of these processes was detected in the brains of both JD-fed SHRSR and JD-fed (SHRsp.SHRsr(D1Rat134-Mt1pa)), both carrying higher levels of brain UCP2 expression (as compared with the other two lines).

Impact of fenofibrate administration on brain UCP2 expression and on stroke occurrence in JD-fed SHRSP. Figure 4 shows the impact of JD plus fenofibrate versus JD alone on brain UCP2 gene and protein expression, and on NF- $K \mathrm{~B}$ and oxidized total protein levels at the end of 4 weeks of treatment. Fenofibrate could restore UCP2 level (panels a and b) and decrease levels of both NF- $\kappa \mathrm{B}$ and oxidative stress (panels c-e).

Figure 5 shows the results of the stroke survival study performed with a long-term fenofibrate administration (3 months) in JD-fed SHRSP. The impact on UCP2 gene and protein expression levels in brains of JD plus fenofibrate treated SHRSP, as compared with animals receiving JD only and JD plus vehicle, is shown in the panels $a$ and $b$ of the Figure 5 . Also at the end of 3 months of treatment, the parallel administration of JD and fenofibrate restored UCP2 level (panels $a$ and b), and decreased levels of both NF- $k B$ and oxidative stress despite the long-term treatment with JD (panels c-e). Importantly, fenofibrate fully protected animals from stroke occurrence over 3 months of follow-up (panel f). In contrast, occurrence of stroke events reached $100 \%$ by the seventh week of JD in both JD and JD plus vehicle treated rats, consistently with previous evidence. ${ }^{3,5}$ SBP and BW values upon fenofibrate administration are reported in the Supplementary Table S1.

Impact of BO administration on brain UCP2 expression and on stroke occurrence in JD-fed SHRSP. Supplementary Figure S1 shows the impact of JD plus BO versus JD alone on brain UCP2 gene and protein expression, NF- $\kappa \mathrm{B}$ and oxidized total protein levels at the end of 4 weeks of treatment. $\mathrm{BO}$ restored UCP2 level (panels a and b) and decreased levels of both NF- $k \mathrm{~B}$ and oxidative stress (panels $c-e$ ).

Figure 6 shows UCP2 gene and protein expression levels, at different experimental times during the stroke survival study, in brains of JD plus BO treated rats, as compared with animals receiving JD only and JD plus BO plus PPAR $a$ inhibitor (panels

Figure 1 Characterization of brain UCP2 gene and protein expression upon JD in SHRSR, SHRSP and in the two SHRSR/SHRSP-STR1/QTL stroke congenic lines (4 weeks of dietary regimen). (a) UCP2 expression in the two parental lines upon either RD or JD; $n=7$ for each line for each treatment. ${ }^{* \star *} P<0.0001$ for JD versus RD fed SHRSP, and for JD-fed SHRSP versus JD-fed SHRSR. (b) WB of UCP2 expression in RD and JD-fed SHRSR, with corresponding densitometric analysis. ${ }^{* * *} P<0.0001$ for JD versus RD. (c) WB of UCP2 expression in RD and JD-fed SHRSP, with corresponding densitometric analysis. ${ }^{* *} P<0.0001$ for JD versus RD. (d) UCP2 expression in the SHRSP-derived stroke congenic line upon RD or JD; $n=6$ for each treatment. (e) WB of UCP2 expression in the SHRSP-derived stroke congenic line upon RD or JD. ${ }^{* *} P<0.001$ for JD versus RD. (f) UCP2 expression in the SHRSR-derived stroke congenic line upon RD or JD. $n=6$ for each treatment. ${ }^{* *} P<0.001$ for JD versus RD. (g) WB of UCP2 expression in the SHRSR-derived stroke congenic line upon RD or JD. ${ }^{*} P<0.001$ for JD versus RD 
a

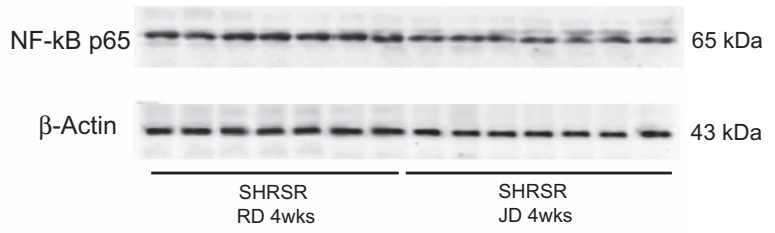

b

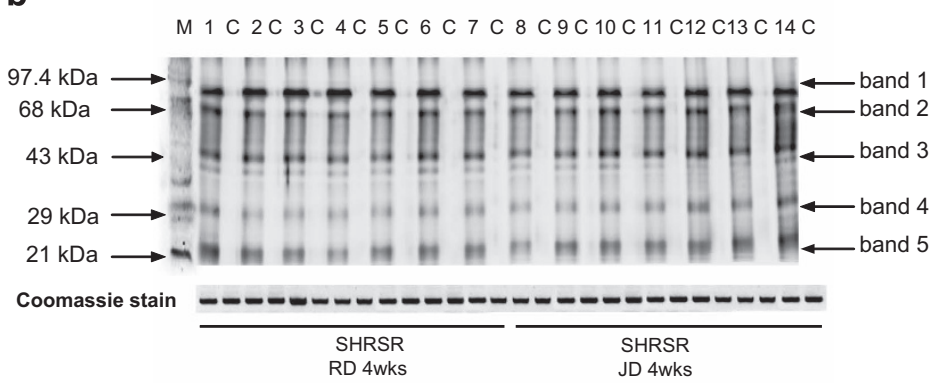

C

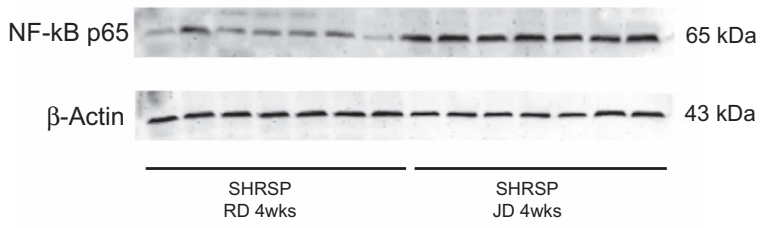

d

M 1 C 2 C 3 C 4 C 5 C 6 C 7 C 8 C 9 C 10 C 11 C 12 C 13 C 14 C

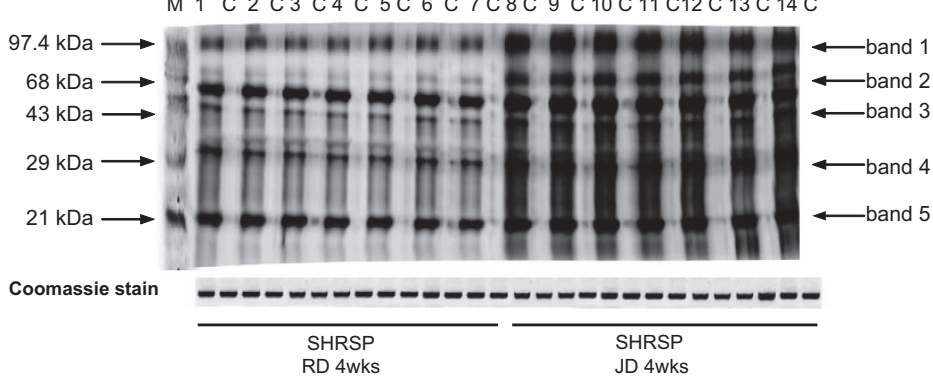

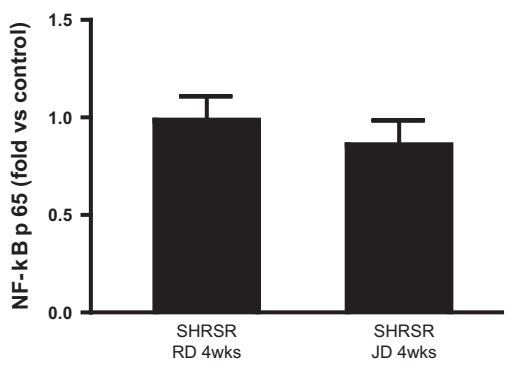
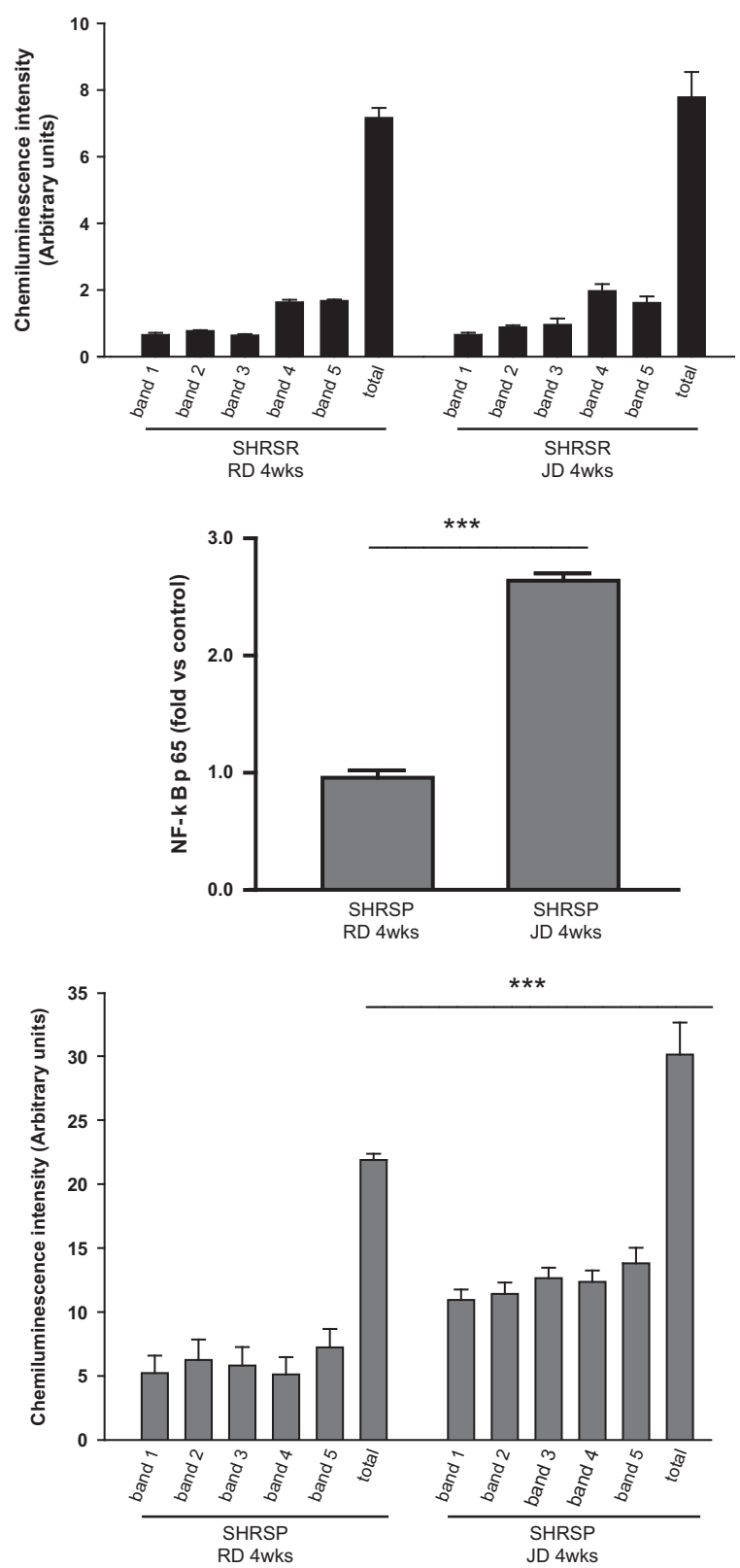

Figure 2 Characterization of NF- $\kappa$ B protein expression and of oxidative stress level in brains of JD-fed SHRSR and SHRSP (4 weeks of dietary regimen). (a) WB of NF- $\kappa B$ expression with corresponding densitometric analysis in SHRSR upon RD or JD. (b) WB of carbonylated total proteins in SHRSR upon RD or JD. Each lane was loaded with $50 \mu \mathrm{g}$ of total proteins. Lane M, DNP marker. Each sample was run with its own untreated control (C). Normalization for lane protein loading was performed using Coomassie staining. The corresponding densitometric analysis is shown on the right side of the panel. Bar graphs represent chemiluminescence intensity relative to the gel loading band. Bands 1 to 5 refer to the most prominent bands on the blots (identified by arrows), whereas total refers to the total chemiluminescence intensity from all bands. (c) WB of NF- $k B$ expression with corresponding densitometric analysis in SHRSP upon RD or JD. ${ }^{* *} P<0.0001$ for JD versus RD. (d) WB of carbonylated total proteins in SHRSP upon RD or JD with corresponding densitometric analysis shown on the right side of the panel. See legend of panel (b). ${ }^{* \star \star} P<0.0001$ for JD versus RD 
a

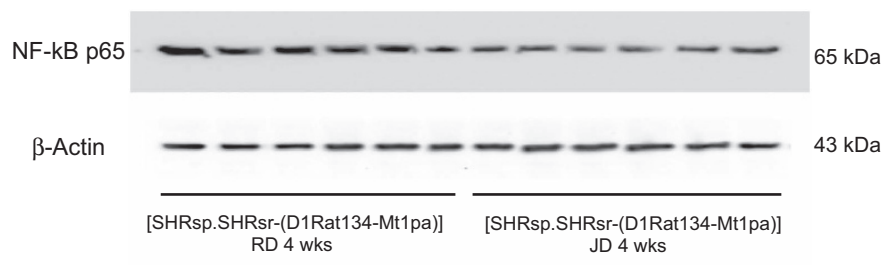

b

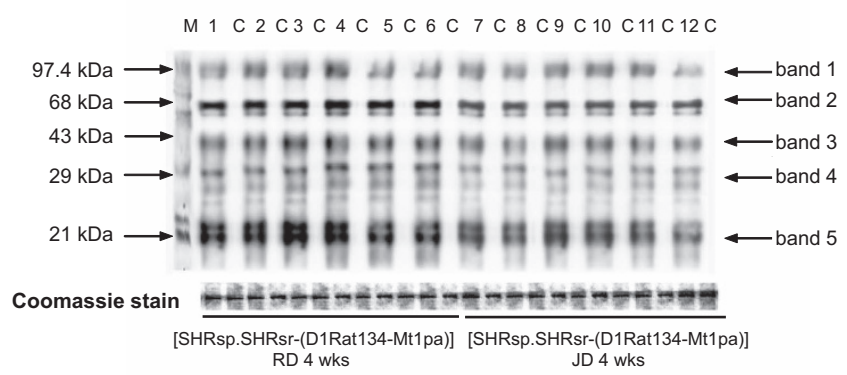

C

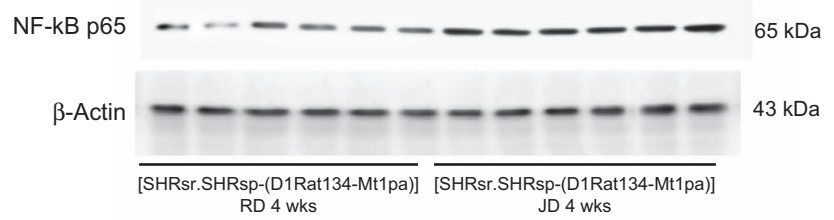

d

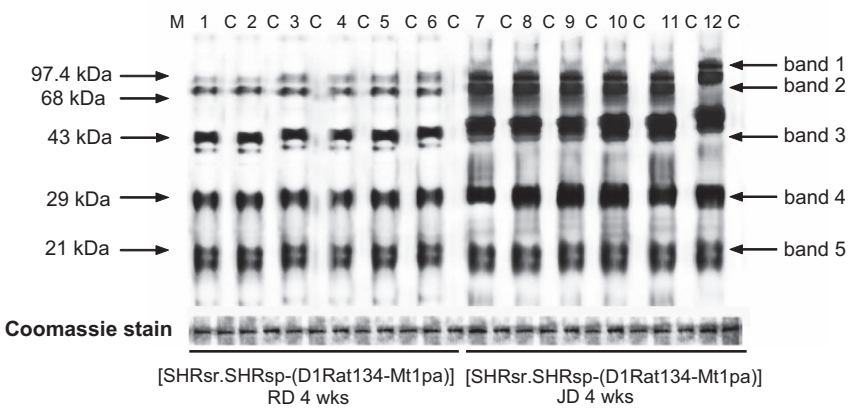

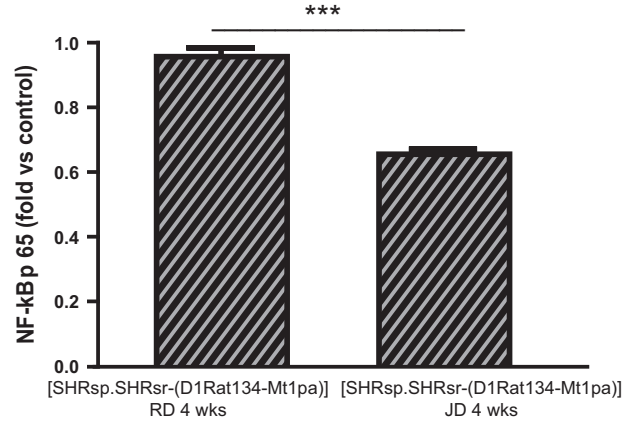
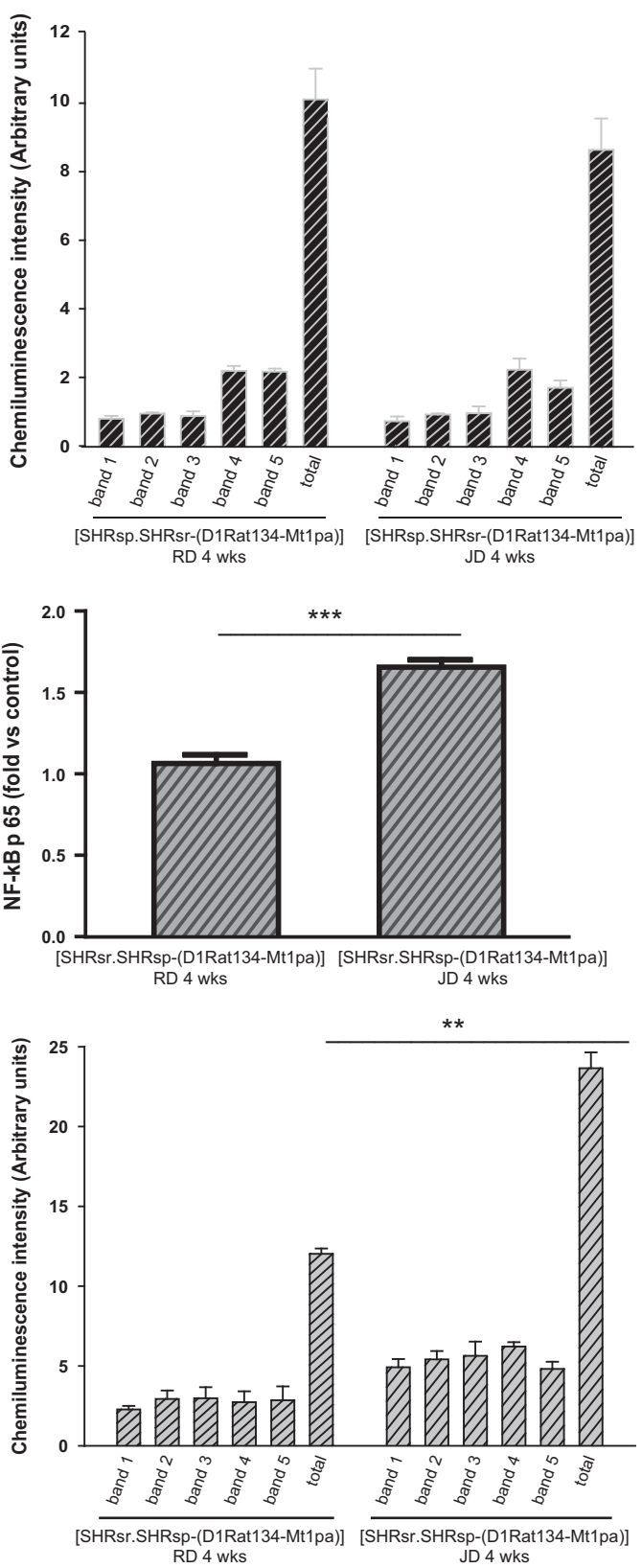

Figure 3 Characterization of NF- $k$ B protein expression and of oxidative stress level in brains of JD-fed SHRSR/SHRSP-STR1/QTL stroke congenic lines (4 weeks of dietary regimen). (a) WB of NF- $\kappa B$ and (b) of carbonylated total proteins in the SHRSP-derived stroke congenic line upon RD or JD. See legend of Figure $2 b$, for the WB of carbonylated total proteins. Bar graphs on the right side represent corresponding densitometric analysis. ${ }^{* \star} P<0.0001$ for JD versus RD. (c) WB of NF- $\kappa$ B and (d) of carbonylated total proteins in the SHRSR-derived stroke congenic line upon RD or JD and corresponding densitometric analysis shown on the right side. See legend of Figure $2 b$ for the WB of carbonylated total proteins. ${ }^{* \star *} P<0.0001$ for JD versus $\mathrm{RD}$. (d) ${ }^{* \star} P<0.001$ for JD versus $\mathrm{RD}$ 
a

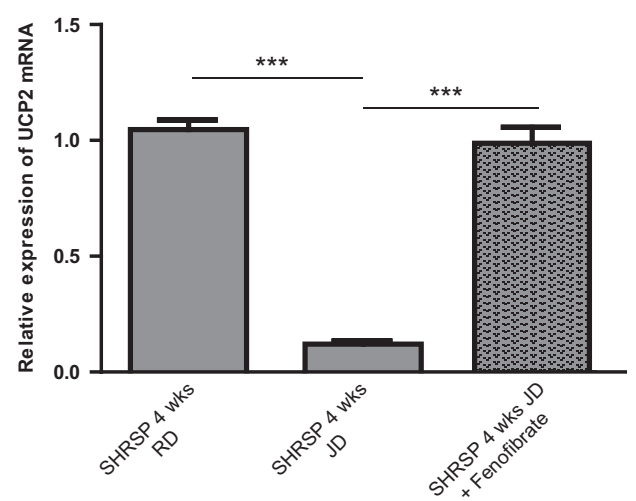

b
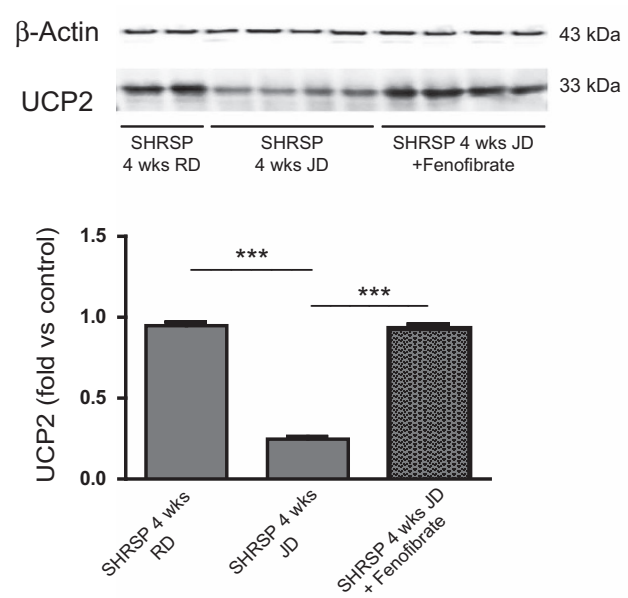

C

NF-kB p65 - -

$\beta$-Actin
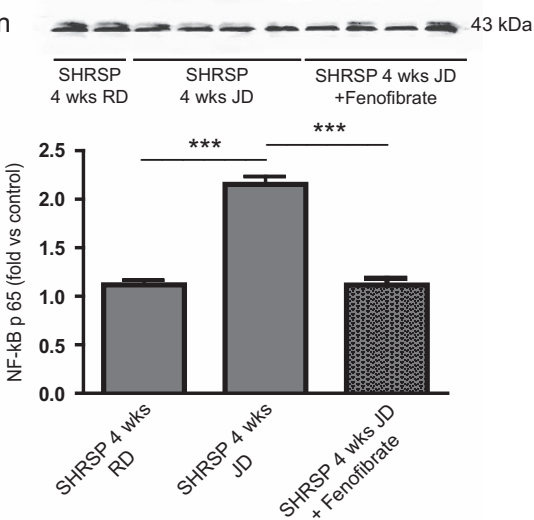

d
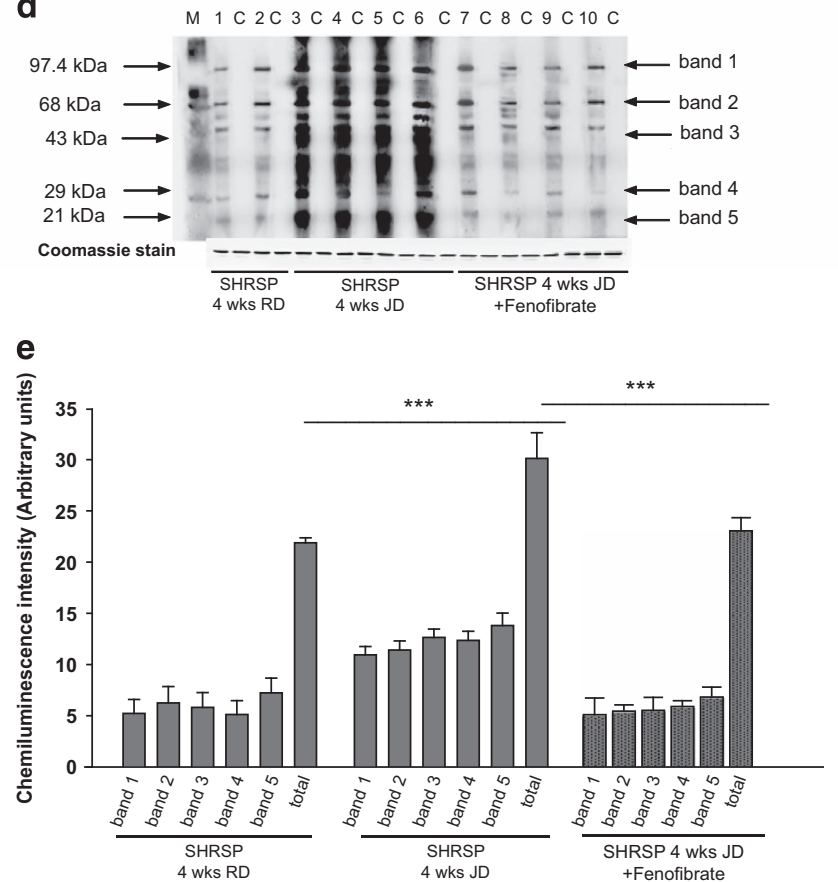

Figure 4 Impact of fenofibrate administration for 4 weeks on brain UCP2 modulation, NF- $\kappa$ B protein expression, oxidative stress level in JD-fed SHRSP. (a and $\mathbf{b}$ ) UCP2 gene and protein expression levels; (c) NF- $\kappa$ B expression level; ( $d$ and e) oxidative stress level; $n=4$ for each experimental group. ${ }^{* \star *} P<0.0001$ for each comparison 
a and $b$ ). As observed at the end of 4 weeks of the combined treatment, the concomitant administration of JD and $\mathrm{BO}$ restored UCP2 level, decreased levels of both NF- $K B$ and oxidative stress despite JD (Figures $6 \mathrm{c}$ and $\mathrm{d}$ and Supplementary Figure S2), and led to a significant delay of stroke occurrence (Figure $6 e$ ). In fact, $40 \%$ of rats survived until the 11th week of treatment. The PPARa inhibitor significantly counteracted the stimulatory effect of the $\mathrm{BO}$ juice on UCP2 expression, therefore leading to $100 \%$ stroke occurrence by the eighth week of treatment (Figures $6 a-e)$, consistently with previous findings. ${ }^{25}$ The SBP and BW values upon these treatments are reported in the Supplementary Table S1.

Analysis of UCP2-targeted microRNAs upon JD versus RD in brains of SHRSR and SHRSP. Out of the compared UCP2-targeted microRNAs in the brains of the SHRSR and SHRSP strains upon the two diets, we detected a remarkable differential expression, very consistent with the parallel differential UCP2 expression, for the rno-microRNA-503. In fact, this miR was remarkably upregulated ( $>2$ folds) in the brain of JD-fed SHRSP whereas it was significantly downregulated in the brain of JD-fed SHRSR as compared with RD (Figure 7a). No other miR showed a significant modulation in relation to the observed UCP2 expression changes. Based on the results of the microRNAs screening, we further explored the modulation of the microRNA-503 expression in our experimental groups. We discovered that SHRSP receiving either fenofibrate or $\mathrm{BO}$ along with JD showed a significant reduction of brain miR-503 expression level (Figures $7 \mathrm{~b}$ and $\mathrm{c}$ ). The expected interference by PPAR $a$ inhibitor was observed in SHRSP receiving JD and $B O$ (Figure 7c). Furthermore, we observed a significant downregulation of brain miR-503 level in the JD-fed SHRSPderived congenic line containing the SHRSR/STR1 fragment (Figure 7d), whereas the SHRSR-derived congenic line, containing the SHRSP/STR1 segment, showed a significant upregulation of miR-503 upon JD (Figure 7e). Therefore, the data obtained in the two STR1/QTL stroke congenic lines reinforced the evidence obtained in the parental lines of origin.

Impact of microRNA-503 overexpression on viability of HUVECs. The in vitro overexpression of hsa-miR-503 in HUVECs showed a marked UCP2 suppression with a linear dose-response (Figures 8a and b). Importantly, at a miR-503 concentration able to turn off UCP2 expression by $90 \%$, a significant increase of cell mortality and a significant decrease of cell viability were observed (Figure $8 \mathrm{c}$ ). The impact on cell viability was comparable to that obtained upon direct UCP2 silencing in HUVECs (Figure 8d).

\section{Discussion}

Our study demonstrates that UCP2 gene and protein expression levels are significantly downregulated by Japanese style dietary feeding in brains of SHRSP but not in brains of its related control strain, the SHRSR. This phenomenon was associated with increased inflammation and oxidative stress. Accordingly, a SHRSR-derived stroke congenic line, carrying a fragment of the SHRSP-STR1/QTL (containing UCP2), showed brain UCP2 downregulation under JD feeding associated with increased inflammation and oxidative stress. Vice versa, brain UCP2 expression did not decrease, and both inflammation and oxidative stress were reduced upon JD in the reciprocal congenic line. Consistently, the administration of JD plus fenofibrate, known to stimulate UCP2 expression, ${ }^{25}$ restored brain UCP2 levels, reduced oxidative stress and fully protected from stroke occurrence the high-salt fed SHRSP. The administration of BO sprouts extract, another stimulator of UCP2 expression, ${ }^{25}$ was also able to delay significantly stroke occurrence in JD-fed SHRSP. The PPARa inhibitor antagonized the beneficial effect of $\mathrm{BO}$, confirming previous evidence obtained in the kidneys. ${ }^{25}$ The greater efficacy of fenofibrate versus BO on stroke protection may be explained by the additional molecular and pharmacological properties of the drug.

Of note, NF- $\mathrm{B}$, which was characterized in the current study mainly for its important role in inflammation, is a ubiquitous transcription factor that, due to its wide range of gene targets, plays several other functions in mammalian cells, particularly in the nervous system. ${ }^{31}$

Based on our results, UCP2 appears to play an important role in the high-salt diet-dependent increased susceptibility to cerebrovascular events, as well as it does for the increased susceptibility to kidney damage of SHRSP. ${ }^{23-25}$ A common molecular mechanism, dependent on UCP2 suppression, may underlie the vascular damage observed in different organs of high-salt-fed SHRSP.

Uncoupling the proton flux through UCP2 is a critical pathway in the regulation of senescence. $8,32,33$ The involvement of UCP2 in vascular diseases is known, being demonstrated in several pathological contexts, ${ }^{9-13}$ and it represents the consequence of its key role in the clearance of ROS within the mitochondria. A neuroprotective effect of UCP2 has been previously associated with its role on oxidative stress. ${ }^{16-21} \mathrm{An}$ association of UCP2 with stroke has been described in other experimental settings. ${ }^{34,35}$

A major strength of our data relies on the evidence obtained in the reciprocal SHRSR/SHRSP-STR1/QTL stroke congenic lines. The stroke phenotype of these lines clearly depends on the genomic configuration of the inserted chromosomal segment belonging to STR1, with evidence that the chromosomal fragment carrying UCP2 significantly interferes with stroke occurrence. ${ }^{5}$ Herein, we report that the introgression of the stroke-prone STR1 chromosomal segment (carrying UCP2) within the stroke-resistant genomic background led to a suppression of UCP2 expression in contrast to the upregulation of the SHRSR strain. The opposite phenomenon was observed in the reciprocal congenic line, supporting the role of the stroke-prone UCP2 configuration to obtain downregulation in response to high-salt diet. We recently reported similar findings on UCP2 gene and protein expression with regard to renal damage in the same stroke congenic lines. ${ }^{24}$

A fundamental demonstration of the role of UCP2 in stroke predisposition of SHRSP was provided by the significant protective impact of $\mathrm{BO}$ and fenofibrate administration, both stimulator of UCP2 expression, ${ }^{25,26}$ toward stroke occurrence despite JD feeding. Of note, our data on fenofibrate as a 
protective agent toward stroke confirm and extend previous findings obtained in the same animal model. ${ }^{30}$

No evidence of UCP2 mutations between the two strains was obtained in our previous study. ${ }^{23}$ In the attempt to unravel, at least in part, some of the mechanisms underlying brain UCP2 downregulation upon JD in the stroke-prone strain, we searched for mechanisms involved in the translational regulation of UCP2. As a result, we found that the UCP2 expression

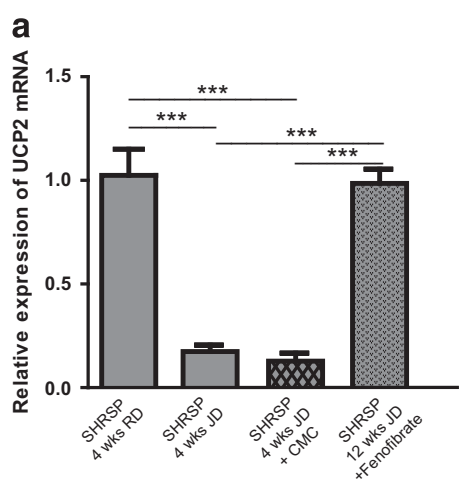

b
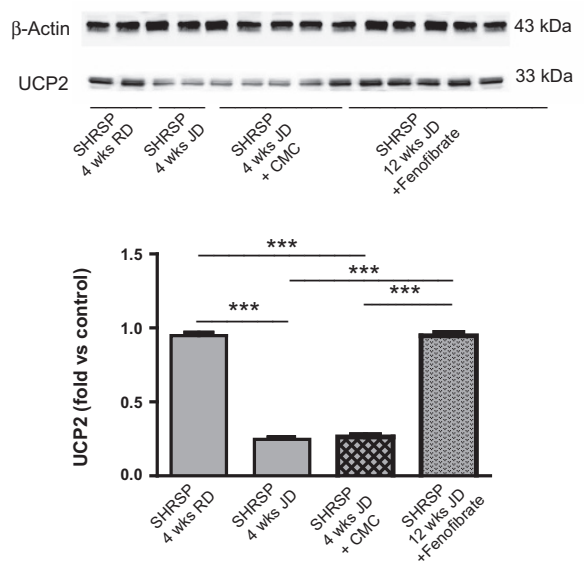

d

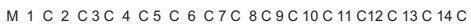

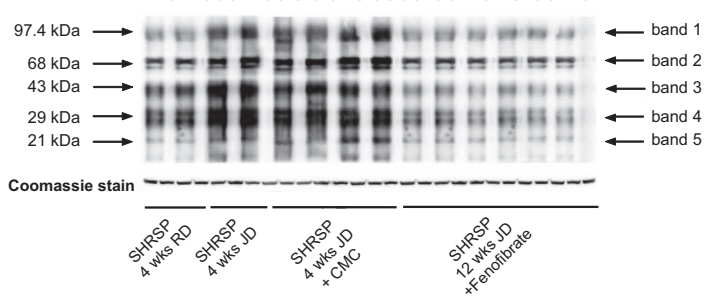

C

NF-kB p65 -

$\beta$-Actin - - - - - - - - $43 \mathrm{kDa}$
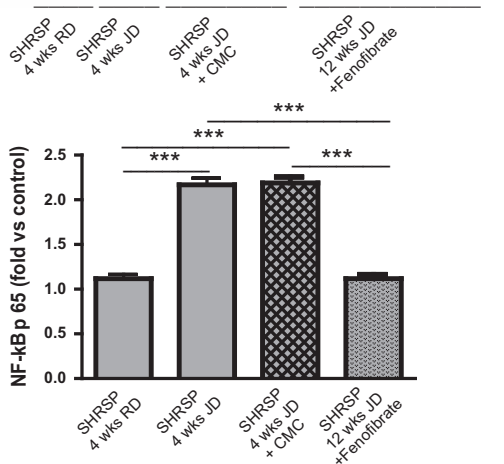

e

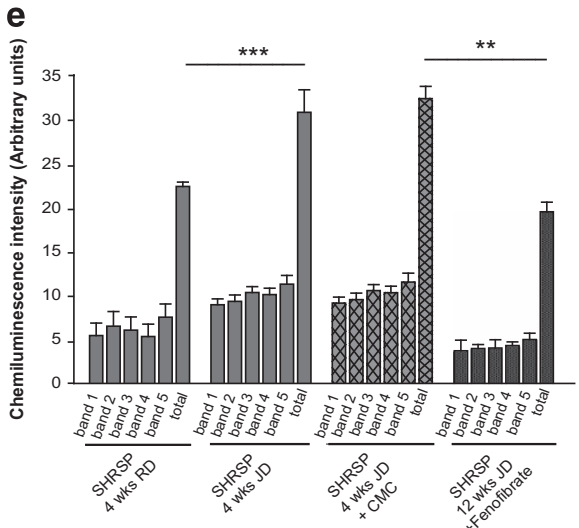

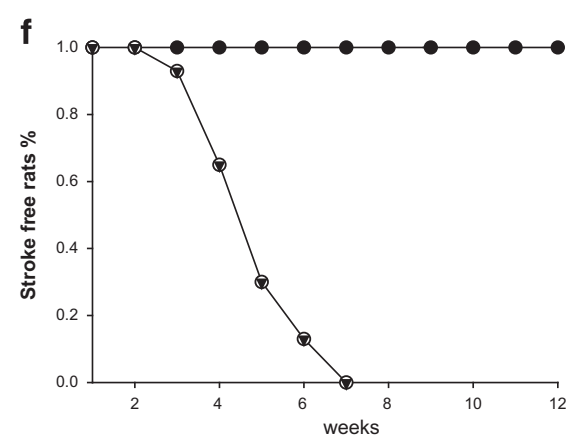

$\begin{array}{ll}- & \text { SHRSP JD +FENOFIBRATE } \\ \text { SHRSP JD +CMC } \\ \text { SHRSP JD }\end{array}$ 
modulation upon JD in the brains of SHRSP and SHRSR was related to the microRNA-503. The latter turned out to be significantly upregulated in high-salt-fed SHRSP, whereas it was significantly decreased in the SHRSR upon the same diet. Consistent results were obtained in the two congenic lines, further supporting the role of this microRNA in UCP2 downregulation. Moreover, treatment with both fenofibrate and BO counteracted the increase of brain microRNA-503 level and the suppression of UCP2 expression in JD-fed SHRSP. Importantly, both treatments, by their ability to restore regular levels of both microRNA-503 and UCP2, significantly protected from stroke occurrence the JD-fed SHRSP. Finally, miR-503 overexpression in vitro abolished UCP2 expression and caused a high degree of cell mortality, consistently with what observed upon direct UCP2 silencing. Our results strongly suggest that miR-503 is a modulator of brain UCP2 expression in high-saltfed SHRSP and also in SHRSR. Of note, miR-503 exerts multiple actions. It is reported as an antiproliferative and antiangiogenetic factor, ${ }^{36}$ and a cell cycle regulator; it is involved in cell adhesion, migration and angiogenesis processes. ${ }^{37}$ Its upregulation in diabetes strongly associates with vascular damage. ${ }^{36,38}$ On the other hand, a decrease of miRNA-503 upon losartan treatment is associated with an improvement of diabetic nephropathy in an animal model of spontaneous type 2 diabetes. ${ }^{39}$ Herein, we report the first evidence that an increase of miR-503 associates with high-salt induced stroke occurrence, through its ability to modulate brain UCP2 expression, in an animal model of spontaneous hypertension and stroke and that, in turn, miR-503 can be decreased by both pharmacological and nutraceutical approaches to obtain protection from stroke. Further studies will address the interaction between PPAR $a$ and miR-503 in the $U C P 2$ regulation. It will be also interesting to characterize the potential contribution of miRNA-503 in the prevention and/or amelioration of hypertensive target organ damage with the available therapeutic antihypertensive strategies.

Our novel findings support the role of microRNAs in the endorgan damage promotion during hypertension. ${ }^{40}$ We are also aware, as a limitation of the current study, that the role of other still unknown UCP2-targeted brain microRNAs remains to be determined in our experimental conditions.

In summary, we demonstrate that UCP2 expression downregulation by high-salt diet associates with increased stroke predisposition whereas UCP2 upregulation, by both nutraceutical and pharmacological agents, associates with a significant stroke protection in high-salt-fed SHRSP. Our data strengthen the role of UCP2 as a suitable therapeutic target for stroke. Notably, the microRNA-503 behaves as a key determinant of the dietary-dependent regulation of UCP2 expression in the brain of SHRSP. Thus, the microRNA-503 has a significant potential in unraveling the mechanisms underlying stroke pathogenesis and may reveal a promising therapeutic agent for this disease.

\section{Materials and Methods \\ Effects of 4 weeks JD feeding on brain UCP2 gene and protein expression in SHRSP, SHRSR and in the SHRSR/SHRSP-STR1/ QTL stroke congenic lines. The following rat lines were used for this study: SHRSP, SHRSR, (SHRsp.SHRsr-(D1Rat134-Mt1pa)), (SHRsr.SHRsp-(D1Rat134- Mt1pa)). The latter two lines are congenic lines carrying the (D1Rat134-Mt1pa) chromosomal segment of STR1 (containing UCP2) in the SHRSR configuration within the SHRSP genomic background (SHRsp.SHRsr-(D1Rat134-Mt1pa)) and, viceversa, in the SHRSP configuration within the SHRSR genomic background (SHRsr.SHRsp-(D1Rat134-Mt1pa)). The preparation of the congenic lines has been previously reported. ${ }^{5}$ By following our standardized experimental protocol, six-week- old male rats of both parental and congenic lines were fed with either RD or JD for 4 weeks (parental lines, $n=7$ for each line at each treatment; congenic lines, $n=6$ for each line at each treatment). At the end of 4 weeks of the dietary treatment, after SBP and BW measurement, animals were killed by cervical dislocation and brains were removed for molecular analyses. Tissue total RNA and proteins were extracted by following previously described procedures. ${ }^{22-25}$ The UCP2 gene and protein expression levels, the $\mathrm{NF}-\kappa \mathrm{B}$ protein expression level and carbonylated total proteins level were assessed by previously described procedures. ${ }^{22-25}$}

Impact of fenofibrate administration on brain UCP2 gene and protein modulation and on stroke occurrence in JD-fed SHRSP. In order to fulfill the first aim, male SHRSP received, starting at 6 weeks of age, JD alone $(n=4)$, JD plus fenofibrate ( $150 \mathrm{mg} / \mathrm{kg} / \mathrm{die}, n=4)$ or vehicle $(1 \% \mathrm{CMC}, n=4)$ administered via gavage for 4 weeks. In order to analyze the impact of fenofibrate on stroke survival, 6-week-old SHRSP received JD alone $(n=6)$, JD plus fenofibrate $(n=6)$ or JD plus CMC $(n=6)$. They were monitored for SBP, BW and stroke occurrence up to 3 months of the dietary plus fenofibrate treatment.

Brains of JD plus fenofibrate treated SHRSP, taken at the end of both 1 month and 3 months of treatment ( $n=4$ and 6 , respectively), were analyzed for UCP2 gene and protein expression levels, NF- $\mathrm{kB}$ protein expression and oxidative stress levels. These molecular analyses were compared with those obtained in brains of 4-week JD-fed SHRSP from the above experimental setup and of 4-week JD plus CMC treated SHRSP.

Impact of BO juice administration on brain UCP2 gene and protein modulation and on stroke occurrence in JD-fed SHRSP. In order to fulfill the first aim, male SHRSP received, starting at 6 weeks of age, JD alone $(n=4)$ or JD plus BO sprouts extract $(n=4)$ for 4 weeks by following previously reported procedures. ${ }^{25}$ In order to explore the impact of BO on stroke survival, male SHRSP received JD alone $(n=6)$, JD plus BO $(n=10)$, JD plus BO plus PPAR $\alpha$ inhibitor $(n=6)$ as previously reported. ${ }^{25} \mathrm{SBP}, \mathrm{BW}$ and stroke occurrence were monitored up to 3 months of the dietary plus $\mathrm{BO}$ juice treatment. Brains of SHRSP, taken either at the end of 4 weeks or at the time of stroke occurrence (between the 8th and 12th week of treatment upon BO; between the 7th and 8th week of treatment upon BO plus PPAR $\alpha$ inhibitor), were analyzed for UCP2 gene and protein expression levels, NF- $\kappa$ B protein expression and oxidative stress levels. These molecular analyses were compared with those obtained in brains of 4-week JD-fed SHRSP from the above experimental setup.

All rats used for the experiments described in the paragraphs 1-3 were maintained at the animal facility of the Neuromed Institution in strict compliance with the guidelines set forth by the American Physiological Society. Animal protocols were approved by the Institutional Animal Care and Use Committee of the Neuromed Institution. Climate was controlled, and temperature was set at $22{ }^{\circ} \mathrm{C}$. Diurnal 12 -h cycles were kept automatically.

Figure 5 Impact of long-term fenofibrate administration on brain UCP2 modulation, NF- $k B$ protein expression, oxidative stress and stroke occurrence in JD-fed SHRSP. (a) UCP2 expression is shown in SHRSP fed for 4 weeks with $\mathrm{RD}(n=7), \mathrm{JD}(n=7)$, JD plus vehicle $(n=4)$ and at the end of three months of JD plus fenofibrate treatment $(n=6) .{ }^{* \star *} P<0.0001$ for each comparison. (b) WB of UCP2 expression in the four experimental groups as above with corresponding densitometric analysis. ${ }^{* \star *} P<0.0001$ for each comparison. (c) WB of NF- $\kappa B$ expression in the four experimental groups as above with corresponding densitometric analysis. ${ }^{* \star *} P<0.0001$ for each comparison. (d) WB of carbonylated total proteins in the four experimental groups with corresponding densitometric analysis (e). See legend of Figure $2 b$, for the WB of carbonylated total proteins. ${ }^{* \star \star} P<0.0001$ for JD versus RD fed SHRSP; ${ }^{\star *} P<0.001$ for JD plus fenofibrate versus JD plus CMC fed SHRSP. (f) Stroke survival rate in the JD, JD plus vehicle and JD plus fenofibrate treated SHRSP. The comparison of JD plus fenofibrate treated SHRSP versus both JD and JD plus vehicle treated SHRSP was significant, $P<0.001$ 
Animals were housed two or three per cage with free access to $\mathrm{RD}$ (containing $22 \%$ protein, $2.7 \mathrm{mg} / \mathrm{g} \mathrm{Na}^{+}, 7.4 \mathrm{mg} / \mathrm{g} \mathrm{K}^{+}, 0.05 \mathrm{mg} / \mathrm{g}$ methionine) and tap water, unless stated otherwise. The JD contained $17.5 \%$ protein, $3.7 \mathrm{mg} / \mathrm{g} \mathrm{K}{ }^{+}$and $0.03 \mathrm{mg} / \mathrm{g}$ methionine (Lab. Piccioni, Milan, Italy), and $1 \% \mathrm{NaCl}$ was added to the drinking water.
Analysis of UCP2-targeted microRNAs expression upon JD versus RD in brains of SHRSR and SHRSP. Based on the very limited knowledge of the rat UCP2 brain modulation by targeted microRNAs, we selected conserved predicted UCP2-targeted microRNAs with all miRSVR scores by

a

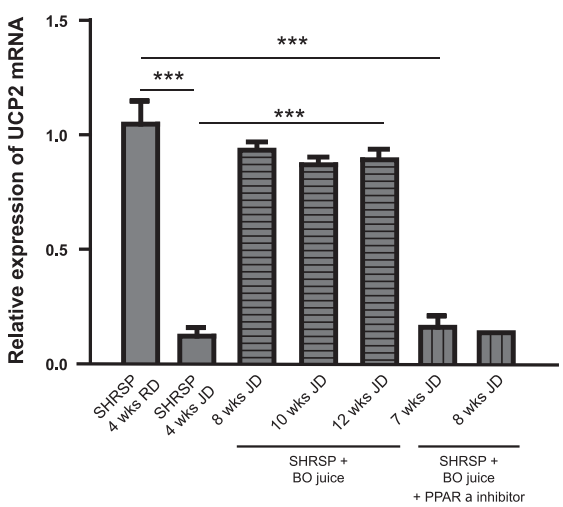

b
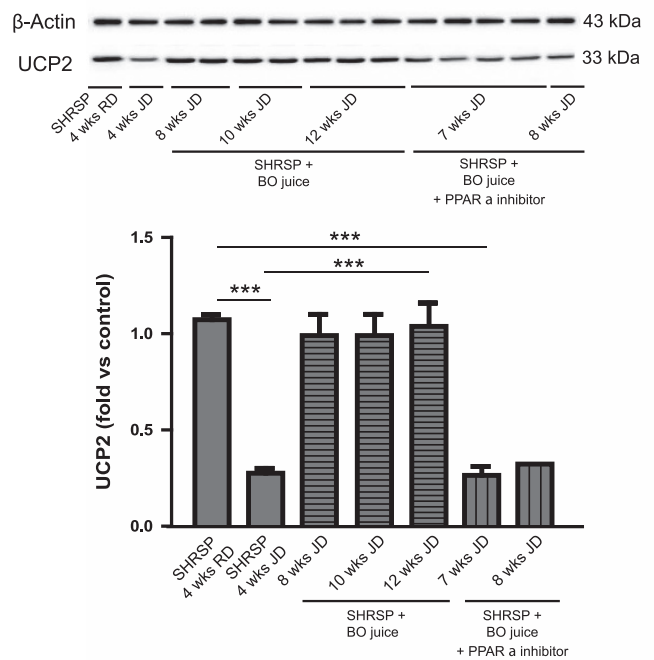

C

NF-kB p65 - - - - - - - - - $65 \mathrm{kDa}$

B-Actin - - - - - - - - - - - $33 \mathrm{kDa}$
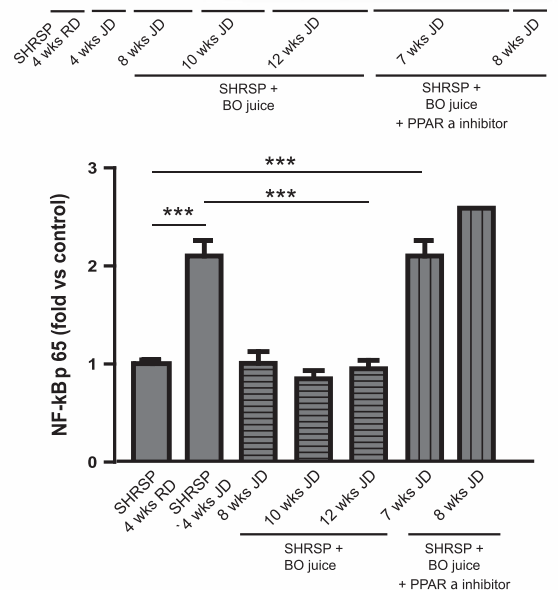

d

M 1 C 2 C 3 C 4 C 5 C 6 C 7 C 8 C 9 C $10 C 11$ C 12 C 13 C 14 C

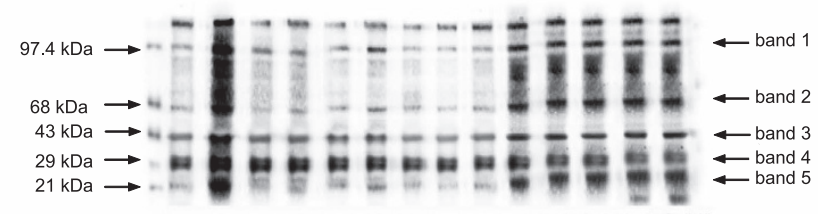

Coomassie stain

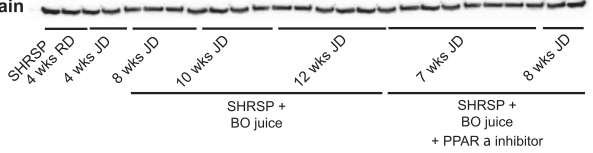

e

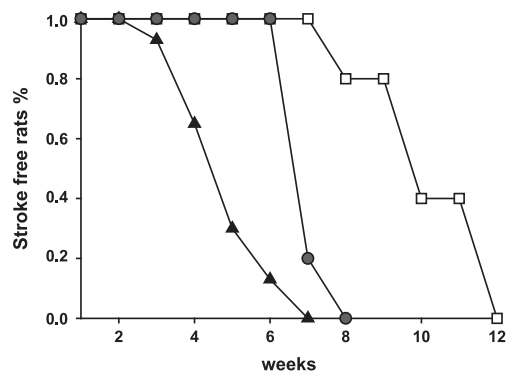

$-\square-$ SHRSP JD+BO juice

$\Delta-$ SHRSPJD

- SHRSP JD+ BO juice+ PPAR a inhibitor 
searching the www.microrna.org public database. The following miRNAs were considered in this study: Rno-microRNA-1, let-7a, let-7b, let-7c, let-7d, let-7i, 16, 24, 27a, 34a, 138, 206, 214, 218, 298, 497, 503. The RT-PCR for each microRNA was performed in triplicate in brain extracts of both parental strains upon the two diets by specific gene expression Taqman assays (Life Technologies). Based on the evidence of a significant microRNA-503 upregulation in the brain of JD-fed SHRSP

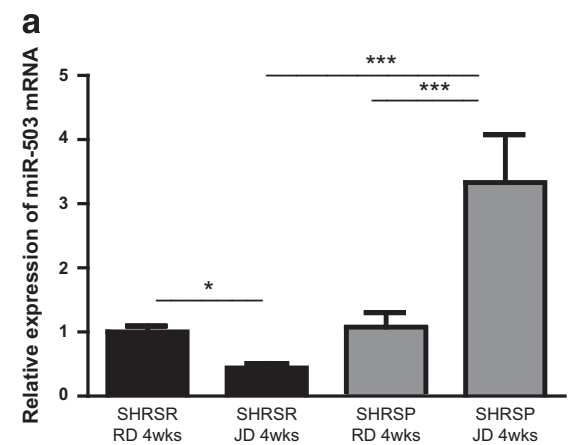

b
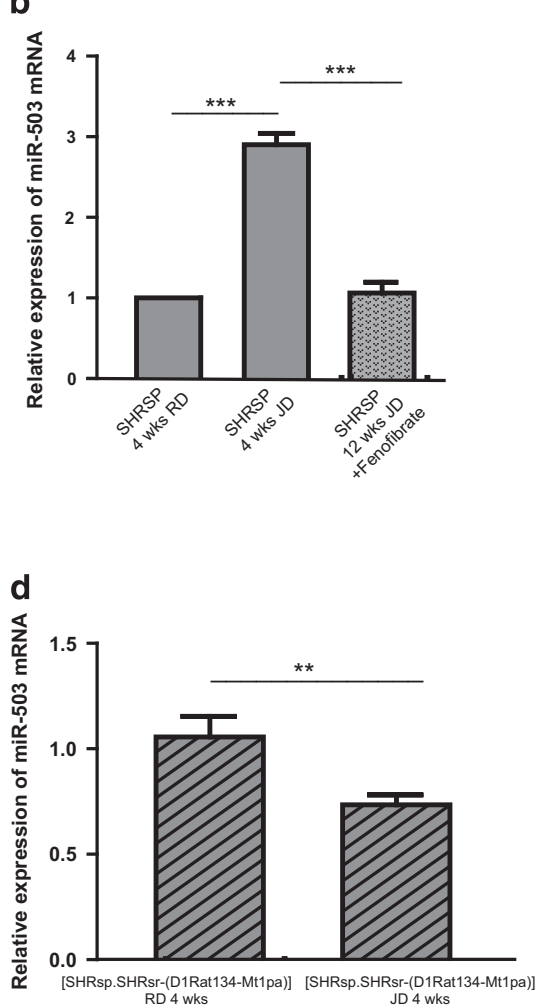
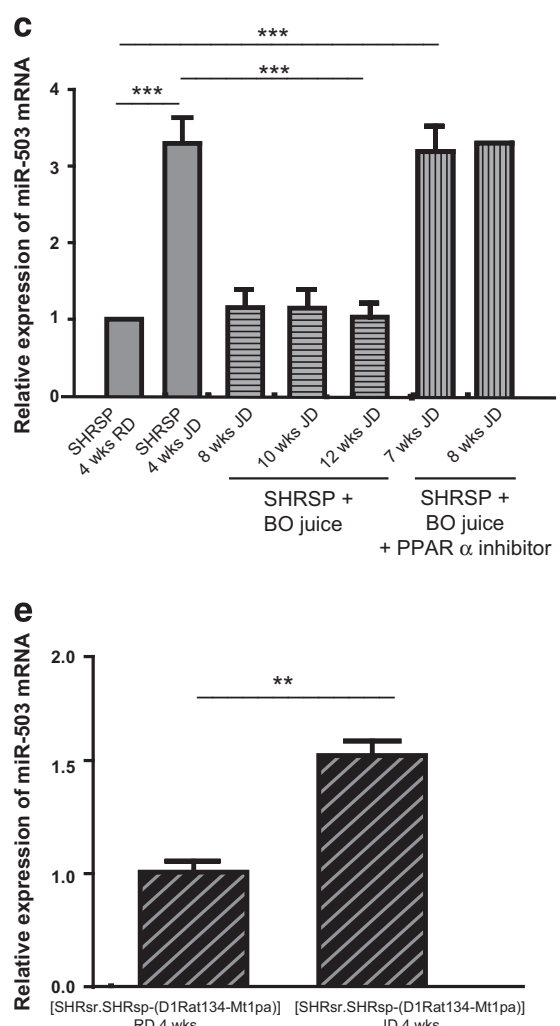

Figure 7 Analysis of brain rno-microRNA-503 expression level in the different experimental conditions. (a) miR-503 level in the SHRSR and SHRSP upon the two diets. ${ }^{* * *} P<0.0001$ for JD versus RD fed SHRSP; ${ }^{* *} P<0.0001$ for JD-fed SHRSP versus JD-fed SHRSR; ${ }^{*} P<0.05$ for JD versus RD fed SHRSR. (b) Impact of fenofibrate administration for 3 months on miR-503 level in JD-fed SHRSP. ${ }^{* \star *} P<0.0001$ for each comparison. (c) Impact of BO alone and of BO plus PPAR $\alpha$ inhibitor administration on miR-503 levels in JD-fed SHRSP. ${ }^{* \star *} P<0.0001$ for each comparison. (d) miR-503 level in the SHRSP-derived stroke congenic line upon the two diets. ${ }^{\star \star} P<0.001$ for JD versus RD. (e) miR-503 level in the SHRSR-derived stroke congenic line upon the two diets. ${ }^{* \star} P<0.001$ for JD versus RD. Rats used for this analysis were the same animals shown in previous Figures $1-6$. For number of animals see the previous figures

Figure 6 Impact of long-term administration of BO on brain UCP2 modulation, NF- $\kappa$ B protein expression, oxidative stress levels and on stroke occurrence in JD-fed SHRSP. (a) UCP2 expression is shown in SHRSP fed for 4 weeks with $\mathrm{RD}(n=7)$, JD $(n=7)$ and in SHRSP receiving both JD plus BO at times of stroke occurrence (8 weeks of treatment, $n=2 ; 10$ weeks, $n=2 ; 12$ weeks, $n=3$ ), SHRSP receiving JD plus BO plus PPAR $\alpha$ inhibitor ( 7 weeks of treatment, $n=4 ; 8$ weeks of treatment, $n=1$ ). ${ }^{* \star \star} P<0.0001$ for each comparison. (b) WB of UCP2 protein expression in the four experimental groups as above with corresponding densitometric analysis. ${ }^{* *} P<0.0001$ for each comparison. (c) WB of NF- $\kappa$ B protein expression in the four experimental groups as above with corresponding densitometric analysis. ${ }^{* \star *} P<0.0001$ for each comparison. (d) WB of carbonylated total proteins in the four experimental groups. See legend of Figure $2 b$, for the WB of carbonylated total proteins. (e) Stroke survival rate in the JD, JD plus BO, JD plus BO plus PPAR $\alpha$ inhibitor treated SHRSP. The comparison of JD plus BO treated SHRSP versus both JD and JD plus BO plus PPAR $\alpha$ inhibitor treated SHRSP was significant, $P<0.001$ and $P<0.001$, respectively 

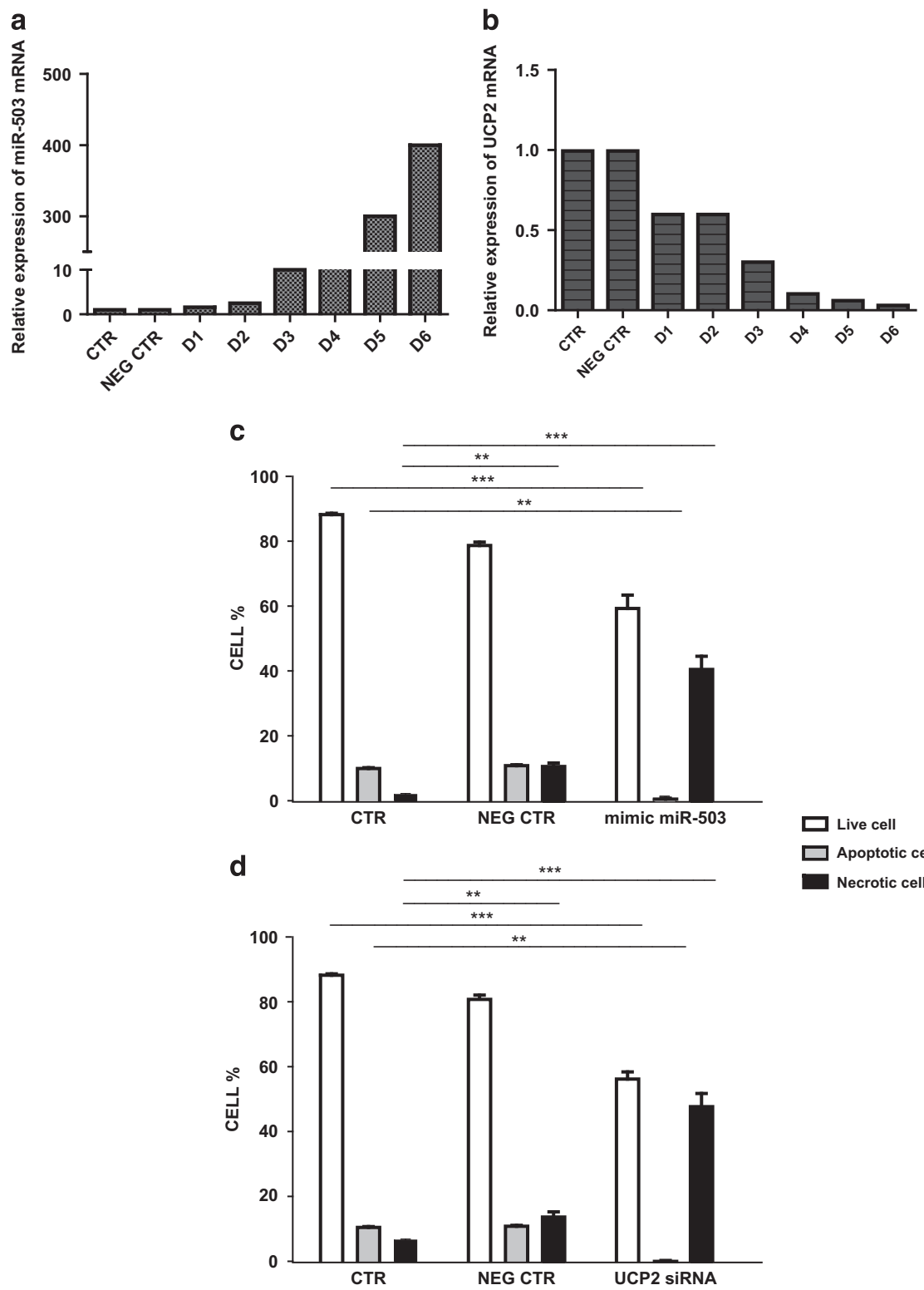

Figure 8 Impact of in vitro overexpression of hsa-microRNA-503 on UCP2 expression and cell viability in HUVECs. (a) miR-503 level in HUVECs transfected with different concentrations of hsa-miR-503. (b) Corresponding UCP2 expression level at each concentration of hsa-miR-503. CTR: control; NEG CTR: cells transfected with lipofectamine and mission miRNA negative control; D1: 12.5 nM; D2: 25 nM; D3: 50 nM; D4: 100 nM; D5: 200 nM; D6: 400 nM hsa-miR-503. (c) FACS analysis of control cells, lipofectaminetreated cells (negative control) and of cells overexpressing miRNA-503 (100 nM). ${ }^{* \star *} P<0.0001$ and ${ }^{\star \star} P<0.001$ for each comparison. (d) FACS analysis of UCP2 silenced cells by using a specific siRNA, compared with both control cells and negative control. ${ }^{* * *} P<0.0001$ and ${ }^{* *} P<0.001$ for each comparison. Each experiment was performed in triplicate. $P=$ NS for comparison of UCP2 siRNA versus mimic hsa-microRNA-503

as opposed to a significant downregulation in the brain of SHRSR (see Results section), the modulation of this miRNA was verified in JD-fed SHRSP upon fenofibrate, vehicle, BO, BO plus PPAR $\alpha$ inhibitor administration, as well as in the brains of the two SHRSR/SHRSP-STR1/QTL stroke congenic lines (by analyzing the same rats used in the above described experimental groups).

In vitro hsa-microRNA-503 overexpression in HUVECs. In order to verify directly the impact of microRNA-503 on UCP2 expression levels, we performed a dose-response experiment in vitro. For this purpose, HUVECs (Lonza, Cambrex, Belgium) were seeded in 60 -mm well plates $\left(2 \times 10^{5} \mathrm{cells} /\right.$ well $)$ and cultured in endothelial growth medium-2 (EGM-2; Lonza) to reach a $70-80 \%$ confluence. Then, serial concentrations of $12.5,25,50,100,200$ and $400 \mathrm{nM}$ of hsa-microRNA-503 mimic (Mission microRNA; Sigma-Aldrich (Milan, Italy)) were incubated in OPTIMEM reduced serum medium with a nucleic acid transferring agent (lipofectamine RNAiMAX reagent (Invitrogen, Milan, Italy)) in a final volume of $2 \mathrm{ml} / \mathrm{well}$ each for $20 \mathrm{~min}$. Five hours later the complex containing medium was replaced with EGM-2 medium supplemented with 10\% fetal bovine serum. Cells transfected with RNAiMax lipofectamine complex and mission miRNA negative control (Sigma-Aldrich) were used as control. Twenty-four hours after transfection cells were extracted for total RNA, by the RNazol procedure, ${ }^{23}$ and used for the evaluation of both miR-503 and UCP2 expression levels by RT-PCR. A specific gene expression Taqman assay (Lifetech, Waltham, MA, USA) was used to assess miR-503 levels, as reported above. The RT-PCR of UCP2 was performed as reported above. Finally, we assessed the impact of miR-503 overexpression at $100 \mathrm{nM}$ concentration (corresponding to $90 \%$ reduction of UCP2 expression) on cell apoptosis, necrosis and viability, as assessed by FACS. The results of FACS were compared with those obtained by performing UCP2 silencing with a specific siRNA in the same cell line (by following previously reported procedures ${ }^{23}$ ). 
Statistical analysis. All values are shown as means + S.E.M. Statistical analysis of SBP, BW, RT-PCR, WB densitometric values and FACS values was performed by one-way ANOVA followed by Bonferroni post hoc test. Comparisons between two groups were performed using Student's t-test followed by post hoc Mann-Whitney test. Survivor function in rats monitored over JD feeding alone, compared with JD plus the different treatments, was estimated by the life-table method. Log-rank and Wilcoxon statistics were used for testing equality of survivor functions.

Statistical significance was stated at the $P<0.05$ level. GraphPad Prism (Ver 5.01 GraphPad Software, Inc., La Jolla, CA, USA) statistical software was used for the statistical analysis.

\section{Conflict of Interest}

The authors declare no conflict of interest.

Acknowledgements. This work was supported by a grant from the Italian Ministry of Health and from the $5 \%$ grant.

1. Rubattu S, Stanzione R, Volpe M. Michondrial dysfunction contributes to hypertensive target organ damage: lessons from an animal model of human disease. Oxid Med Cell Longev 2016; 2016: 1067801.

2. Volpe M, Camargo M, Mueller F, Campbell WJ, Sealey J, Pecker M et al. Relation of plasma renin to end organ damage and to protection of $\mathrm{K}$ feeding in stroke-prone hypertensive rats. Hypertension 1990; 15: 318-326.

3. Rubattu S, Volpe M, Kreutz R, Ganten U, Ganten D, Lindpaintner K. Chromosomal mapping of quantitative trait loci contributing to stroke in a rat model of complex human disease. Nat Genet 1996; 13: 429-434.

4. Schreiber S, Bueche CZ, Garz C, Kropf S, Kuester D, Amann K et al. Kidney pathology precedes and predicts the pathological cascade of cerebrovascular lesions in stroke prone rats. PLOS ONE 2011; 6: e26287.

5. Rubattu S, Hubner N, Ganten U, Evangelista A, Stanzione R, Di Angelantonio E et al. Reciprocal congenic lines for a major stroke QTL on rat chromosome 1. Physiol Genomics 2006; 27: 108-113.

6. Mattiasson G, Sullivan PG. The emerging functions of UCP2 in health, disease, and therapeutics. Antioxid Redox Signal 2006; 8: 243-270.

7. Villarroya $F$, Iglesias $R$, Giralt $M$. PPARs in the control of uncoupling proteins gene expression. PPAR Res 2007; 2007: 74364

8. Israelian-Konaraki Z, Reaven PD. Peroxisome proliferator-activated receptor-alpha and atherosclerosis: from basic mechanisms to clinical implications. Cardiology 2005; 103: 1-9.

9. Andrews ZB, Horvath TL. Uncoupling protein-2 regulates lifespan in mice. Am J Physiol Endocrinol Metab 2009; 296: E621-E627.

10. Ma S, Ma L, Yang D, Luo Z, Hao X, Liu D et al. Uncoupling protein 2 ablation exacerbates high-salt intake-induced vascular dysfunction. Am J Hypertens 2010; 23: 822-828.

11. Ma S, Zhang Y, Wang Q, Yang D, Li D, Tang B et al. Ablation of uncoupling protein 2 exacerbates salt-induced cardiovascular and renal remodeling associated with enhanced oxidative stress. Int J Cardiol 2014; 175: 206-210.

12. Moukdar F, Robidoux J, Lyght O, Pi J, Daniel KW, Collins S. Reduced antioxidant capacity and diet-induced atherosclerosis in uncoupling protein-2-deficient mice. J Lipid Res 2009; 50: 59-70.

13. Tian $X Y$, Wong $W T, X u A$, Lu $Y$, Zhang $Y$, Wang $L$ et al. Uncoupling protein-2 protects endothelial function in diet-induced obese mice. Circ Res 2012; 110: 1211-1216.

14. Ma S, Wang Q, Zhang Y, Yang D, Li D, Tang B et al. Transgenic overexpression of uncoupling protein 2 attenuates salt-induced vascular dysfunction by inhibition of oxidative stress. Am J Hypertens 2014; 27: 345-354.

15. Richard D, Clavel S, Huang Q, Sanchis D, Ricquier D. Uncoupling protein 2 in the brain distribution and function. Biochem Soc Trans 2001; 29: 812-817.

16. Hass DT, Barnstable CJ. Uncoupling protein 2 in the glial response to stress: Implications for neuroprotection. Neural Regen Res 2016; 11: 1197-1200.

17. Mattiasson G, Shamloo M, Gido G, Mathi K, Tomasevic G, Yi S et al. Uncoupling protein-2 prevents neuronal death and diminishes brain dysfunction after stroke and brain trauma. Nat Med 2003; 9: 1062-1068.

18. Normoyle KP, Kim M, Farahvar A, Llano D, Jacson K, Wang H. The emerging neuroprotective role of mitochondrial uncoupling protein-2 in traumatic brain injury. Transl Neurosci 2015; 6: 179-186.

19. Haines B a, Mehta SL, Pratt SM, Warden CH, Li PA. Deletion of mitochondrial uncoupling protein-2 increases ischemic brain damage after transient focal ischemia by altering gene expression patterns and enhancing inflammatory cytokines. J Cereb Blood Flow Metab 2010; 30: 1825-1833.

20. Lapp DW, Zhang SS, Barnstable CJ. Stat3 mediates LIF-induced protection of astrocytes against toxic ROS by upregulating the UCP2 mRNA pool. Glia 2014; 62: 159-170.
21. Ho PW, Liu HF, Ho JW, Zhang WY, Chu AC, Kwok KH et al. Mitochondrial uncoupling protein-2 (UCP2) mediates leptin protection against MPP+ toxicity in neuronal cells. Neurotox Res 2010; 17: 332-343.

22. Rubattu S, Bianchi F, Busceti CL, Cotugno M, Stanzione R, Marchitti S et al. Differential modulation of AMPK/PPAR $\alpha / \mathrm{UCP} 2$ axis in relation to hypertension and aging in the brain, kidneys and heart of two closely related spontaneously hypertensive rat strains. Oncotarget 2015; 6: 18800-18818.

23. Di Castro S, Scarpino S, Marchitti S, Bianchi F, Stanzione R, Cotugno M et al. Differential modulation of uncoupling protein 2 in kidneys of stroke-prone spontaneously hypertensive rats under high-salt/low-potassium diet. Hypertension 2013; 61: 534-541.

24. Rubattu S, Cotugno M, Bianchi F, Sironi L, Gelosa P, Stanzione R et al. A differentia expression of Uncoupling Protein 2 associates with renal damage in stroke-resistant spontaneously hypertensive rat/stroke-prone spontaneously hypertensive rat-derived stroke congenic lines. J Hypertens 2017 (in press).

25. Rubattu S, Di Castro S, Cotugno M, Bianchi F, Mattioli R, Baima S et al. Protective effects of Brassica oleracea sprouts extract toward renal damage in high-salt-fed SHRSP: role of AMPK/PPAR $\alpha / U C P 2$ axis. J Hypertens 2015; 33: 1465-1479.

26. Srivastava RAK, Jahagirdar R, Azhar S, Sharma S, Bisgaier CL. Peroxisome proliferatoractivated receptor-alpha selective ligand reduces adiposity, improves insulin sensitivity and inhibits atherosclerosis in LDL receptor-deficient mice. Mol Cell Biochem 2006; 285: 35-50.

27. Park CW, Zhang Y, Zhang X, Wu J, Chen L, Cha DR et al. PPARalpha agonist fenofibrate improves diabetic nephropathy in db/db mice. Kidney Int 2006; 69: 1511-1517.

28. Deplanque D, Gelé P, Pétrault O, Six I, Furman C, Bouly M et al. Peroxisome-proliferatoractivated receptor-alpha activation as a mechanism of preventive neuroprotection induced by chronic fenofibrate treatment. J Neurosci 2003; 23: 6264-6271.

29. Wang G, Liu X, Guo Q, Namura S. Chronic treatment with fibrates elevates superoxide dismutase in adult mouse brain microvessels. Brain Res 2010; 1359: 247-255.

30. Gelosa P, Banfi C, Gianella A, Brioschi M, Pignieri A, Nobili E et al. Peroxisome proliferatoractivated receptor \{alpha\} agonism prevents renal damage and the oxidative stress and inflammatory processes affecting the brains of stroke-prone rats. J Pharmacol Exp Ther 2010; 335: 324-331.

31. O'Neill LA, Kaltschmidt C, NF-kappa B. a crucial transcription factor for glial and neuronal cell function. Trends Neurosci 1997; 20: 252-258.

32. Dikov D, Aulbach A, Muster B, Dröse S, Jendrach M, Bereiter-Hahn J. Do UCP2 and mild uncoupling improve longevity? Exp Gerontol 2010; 45: 586-595.

33. Dietrich MO, Horvath TL. The role of mitochondrial uncoupling proteins in lifespan. Pflugers Arch Eur J Physiol 2010; 459: 269-275.

34. Pu Y, Zhang H, Wang P, Zhao Y, Li Q, Wei X et al. Dietary curcumin ameliorates agingrelated cerebrovascular dysfunction through the ampk/uncoupling protein 2 pathway. Cell Physiol Biochem 2013; 32: 1167-1177.

35. Della-Morte D, Dave KR, DeFazio RA, Bao YC, Raval AP, Perez-Pinzon MA. Resveratro pretreatment protects rat brain from cerebral ischemic damage via a sirtuin 1-uncoupling protein 2 pathway. Neuroscience 2009; 159: 993-1002.

36. Caporali A, Meloni M, Völlenkle C, Bonci D, Sala-Newby GB, Addis R et al. Deregulation of microRNA-503 contributes to diabetes mellitus-induced impairment of endothelial function and reparative angiogenesis after limb ischemia. Circulation 2011; 123: 282-291.

37. Caporali A, Emanueli C. MicroRNA-503 and the extended MicroRNA-16 family in angiogenesis. Trends Cardiovasc Med 2011; 21: 162-166.

38. Caporali A, Meloni M, Nailor A, Mitić T, Shantikumar S, Riu F et al. p75(NTR)-dependent activation of NF-KB regulates microRNA-503 transcription and pericyte-endothelial crosstalk in diabetes after limb ischaemia. Nat Commun 2015; 6: 8024.

39. Zhu X, Zhang $C$, Fan $Q$, Liu X, Yang G, Jiang $Y$ et al. Inhibiting MicroRNA-503 and MicroRNA-181d with Losartan ameliorates diabetic nephropathy in KKAy mice. Med Sci Monit 2016; 22: 3902-3909.

40. Heggermont WA, Heymans S. MicroRNAs are involved in end-organ damage during hypertension. Hypertension 2012; 60: 1088-1093.

Cell Death and Disease is an open-access journal published by Nature Publishing Group. This work is licensed under a Creative Commons Attribution 4.0 International License. The images or other third party material in this article are included in the article's Creative Commons license, unless indicated otherwise in the credit line; if the material is not included under the Creative Commons license, users will need to obtain permission from the license holder to reproduce the material. To view a copy of this license, visit http://creativecommons.org/licenses/by/4.0/

(C) The Author(s) 2017 